\title{
Evaluation and Selection of Manufacturing Suppliers in B2B E-Commerce Environment
}

\author{
Quan Zhang (D), Zhen Guo (iD, Feiyu Man (D), and Jiyun Ma (iD \\ School of Information Engineering, ShenYang University of Technology, ShenYang 110870, China \\ Correspondence should be addressed to Quan Zhang; isqzhang@sut.edu.cn
}

Received 1 May 2020; Revised 24 May 2020; Accepted 1 June 2020; Published 22 June 2020

Guest Editor: Lei Xie

Copyright ( 92020 Quan Zhang et al. This is an open access article distributed under the Creative Commons Attribution License, which permits unrestricted use, distribution, and reproduction in any medium, provided the original work is properly cited.

\begin{abstract}
The evaluation and selection of manufacturing suppliers in B2B e-commerce environment is summed up as a multiple-attribute decision-making problem. In B2B E-commerce environment, some performance indicators of manufacturing suppliers present uncertainty and could not be expressed with precise numeric values. Linguistic terms, preference orderings, or interval numbers are commonly used to express the performances of the suppliers accurately instead of crisp values when the available information is uncertain or incomplete. This paper proposes an approach to the selection of manufacturing suppliers in B2B E-commerce environment, where the attribute values in decision matrix are expressed with linguistic terms, preference orderings, and interval numbers. Firstly, the hybrid decision matrix is normalized by calculating the grey correlation coefficients of attribute values with the ideal values of attributes. Secondly, a deviation maximization model is proposed to determine the attribute weights, which is combined with those derived from the entropy method. Thirdly, the overall values of suppliers are calculated and their rankings are obtained. Finally, an example is used to illustrate the proposed approach.
\end{abstract}

\section{Introduction}

As important components of the supply chain, suppliers usually play important roles in the manufacturing process [1-3]. The relationships between manufacturers and suppliers are examined by Svensson et al. [2]. The evaluation and selection of suppliers are important steps in the operations of manufacturers and can be modeled as multipleattribute decision-making (MADM) problems, which involve some qualitative attributes, for example, the quality factor and risk factor of the suppliers.

Traditionally, both the qualitative attributes and the quantitative attributes are adopted in modeling the evaluation and selection of manufacturing suppliers [4]. However, in B2B E-commerce environment, both the qualitative attributes and the quantitative attributes of the suppliers show much more ambiguity and uncertainty than before $[5,6]$. Natural language is introduced by Zadeh [7] and can be used to reduce the burden of expressing subjective uncertain judgments in a decision-making process. A linguistic term is one of the easy ways for evaluations in uncertain environment. Linguistic terms are usually used to assess the qualitative attributes of the suppliers $[4,8]$. In the meantime, because the information available is uncertain or incomplete, preference orderings are also likely to be employed to evaluate the qualitative attributes, in addition to linguistic terms. Preference orderings are also the easy ways for evaluating the suppliers by describing their relative positions, i.e., the ranking orders of the suppliers against some qualitative attributes. Preference orderings are used to evaluate the attribute weights in [9].

Furthermore, in the global competitive situations, against the quantitative attributes, the performance of suppliers would be a range of possible values, with the minimum and the maximum of the utility scores [9]. In other words, interval numbers are the ways of evaluating suppliers against some quantitative attributes [9].

It can be seen that, in B2B E-commerce environment, linguistic terms, preference orderings, and interval numbers are the appropriate expressions of the performance indicators (i.e., attribute values) of manufacturing suppliers. In this case, we are facing challenges when evaluating and 
selecting the suppliers for the manufacturers in $\mathrm{B} 2 \mathrm{~B}$ E-commerce environment. It is desirable to propose an approach to the evaluation and selection of manufacturing suppliers in B2B E-commerce environment when their performance indicators are expressed by means of such hybrid information.

However, the research on evaluating the manufacturing suppliers in B2B E-commerce environment is not so common when their performances or attribute values are multiple types of information, such as linguistic terms, preference orderings, and interval numbers. The purpose of this paper is to develop an approach for evaluating and selecting the manufacturing suppliers in $\mathrm{B} 2 \mathrm{~B}$ E-commerce environment, where their attribute values are expressed with linguistic terms, preference orderings, and interval numbers. Normalizations on the hybrid attribute values are conducted, based on which the attribute weights are determined so that their overall performances (overall values) are obtained for rankings and selections.

\section{Current Research and Research Objectives}

The approaches of evaluating and selecting suppliers can be classified into three categories, including (i) multiple attribute decision-making approaches [9-12], (ii) mathematical programming approaches [13-15], and (iii) intelligent approaches (e.g., ANN and Grey system theory).

According to the multiple-attribute decision-making approaches, linear weighting methods and TOPSIS are usually utilized. With linear weighting methods, the overall values of every suppliers are calculated by summing up every attribute values multiplied by their corresponding weights. The supplier(s) with the highest overall values would be selected. TOPSIS, i.e., the technique for order preference by similarity to an ideal solution, was first proposed by Hwang and Yoon [16]. The TOPSIS method ranks a limited number of alternatives according to the relative degree of proximity to the idealized alternatives [10-12]. The best the alternative is the smallest degree of proximity to the idealized alternatives. Generally, when applying the linear weighting methods and TOPSIS, attribute weights should be determined beforehand, and the attribute weights can be assigned by decision makers or by using the AHP method subjectively.

According to the mathematical programming approaches, usually, multiple objectives are contained in the objective function, as well as some constraints are considered simultaneously. In [13], in order to determine the best suppliers, AHP is integrated with nonlinear and multiobjective integer programming model, under quantity discounts and capacity and budget constraints, while the objectives of the model are maximizing the total value of purchase (TVP), minimizing the total cost of purchase (TCP), or maximizing TVP and minimizing TCP simultaneously. In [14], a mixed-integer nonlinear program is proposed to solve dynamic supplier selection problems. In [15], the problem of supplier selection and order allocation with multiperiod, multiproduct, multisupplier, and multiobjective is generalized as a mixed integer linear programming model, where the objectives are total inventory cost (i.e., delay, holding and shortage, ordering, and discounted purchase costs) and the constraints are the budget and capacity limitations for both buyers and suppliers. The model is solved by means of a preemptive fuzzy goal programming approach.

According to the intelligent approaches, artificial neural network (ANN) models $[17,18]$ and Grey theory [19-21] are usually employed. In [17], an artificial neural network-based predictive model is developed for forecasting the supplier's bid prices in the supplier selection negotiation process, by allowing a demander to foresee the relationship between its alternative bids and corresponding supplier's next bid prices in advance, which decreases the meaningless negotiation times, reduces the procurement cost, improves the negotiation efficiency, or shortens the supplier selection lead-time. In [18], an adaptive neuro-fuzzy inference system is developed by determining the criteria and applied for supplier selections.

In the recent years, grey theory is applied to deal with uncertainty inherent in evaluating the suppliers while the linguistic terms are adopted to express their attribute values [19-21]. Although the proposed approach in [21] does not require any probability distribution or fuzzy membership function, preference orderings and interval numbers are not considered for the attribute values of suppliers.

In [9], the TOPSIS method is extended to the situation by allowing the input attribute values being interval numbers with the minimum and the maximum of the utility scores (a range of possible values for quantitative attributes and a list of possible grades for qualitative attributes). The rank order centroid (ROC) method is used to determine the attribute weights based on the attribute ranking orders [22, 23]. The minimum and the maximum outputs of the extended TOPSIS are obtained, and their averages are adopted as the overall index for selections.

There is rare research on tackling the supplier selection problems with the attribute values being linguistic terms, preference orderings, and interval numbers. The research objective of this paper is to propose a new approach to deal with the qualitative attribute values expressed with linguistic terms and preference orderings and the quantitative attribute values expressed with interval numbers, when evaluating and selecting the manufacturing suppliers in B2B E-commerce environment.

This paper is organized as follows. Section 1 introduces the research background. Section 2 reviews the current research on evaluating and selecting suppliers. Section 3 describes the evaluation and selection of suppliers as a MADM problem. In Section 4, a new approach is proposed to evaluating and selecting the manufacturing suppliers in B2B E-commerce environment, while the attribute values are expressed in linguistic terms, preference orderings, and interval numbers. In Section 5, an example is used to illustrate the proposed approach. Section 6 gives the conclusions and discussions.

\section{Problem Descriptions}

As stated above, the evaluation and selection of manufacturing suppliers in B2B E-commerce environment is modeled as a MADM problem. The following notations 
and assumptions are used to represent the MADM problem of evaluating and selecting manufacturing suppliers.

The alternatives (i.e., the suppliers) are known. Let $S=$ $\left\{S_{1}, S_{2}, \ldots, S_{m}\right\}$ denote a discrete set of $m(\geq 2)$ possible alternatives. The attributes are known, and let $C=\left\{C_{1}, C_{2}, \ldots, C_{n}\right\}$ denote a set of $n(\geq 2)$ attributes. In order to distinguish the qualitative and quantitative attributes with different characteristics, the subscript of the attributes is divided into three categories: $J_{1}$ for the subscript set of attributes with linguistic assessment values, $J_{2}$ for the subscript set of attributes with preference ordering assessment values, and $J_{3}$ for the subscript set of attributes with interval number assessment values.

Let $\widetilde{A}=\left[\widetilde{a}_{i j}\right]_{m \times n}$ denote the decision matrix, where $\widetilde{a}_{i j}$ are the assessment values for alternative $S_{i}$ with respect to attribute $C_{j}, i=1, \ldots, m$, and $j=1, \ldots, n$. In this study, $\widetilde{a}_{i j}$ are in the forms of linguistic terms, preference orderings, and interval numbers.

Let $W=\left(w_{1}, w_{2}, \ldots, w_{n}\right)$ denote the weight vector of $n(\geq 2)$ attributes, where $w_{j}$ is the weight of attribute $C_{j}$, while $\sum_{j=1}^{n} w_{j}=1$ and $w_{j}>0$ holds for $j=1, \ldots, n$.

The problem focused in this paper is to select the best supplier(s) for a manufacturer in B2B E-commerce environment, while their performance indicators (i.e., attribute values) are linguistic terms, preference orderings, and interval numbers.

\section{The Proposed Approach}

The proposed approach to the problem stated in Section 3 is composed of three steps: normalize the attribute values in different formats based on the grey relational degree method, determine the attribute weights, and calculate the overall values of the alternatives (suppliers).

4.1. Normalize the Attribute Values in Different Formats. Since the attribute values in decision matrix $\widetilde{A}=\left[\widetilde{a}_{i j}\right]_{m \times n}$ are in the formats of linguistic terms, preference orderings, and interval numbers, corresponding methods are developed for transforming them into a comparable format, i.e., the utility value.

\subsubsection{Calculate the Grey Correlation Coefficients of Linguistic Attribute Values}

Definition 1. A linguistic term $\widetilde{T}$ on a real-number set is defined as a triangular fuzzy number (denoted as $(u, \alpha$, and $\beta)$ ), if its membership function $\mu_{T}\left(R^{+} \longrightarrow[0,1]\right)$ is defined as

$$
\mu_{T}(x)= \begin{cases}\frac{x-\alpha}{u-\alpha}, & x \in[\alpha, u], \\ \frac{x-\beta}{u-\beta}, & x \in[u, \beta], \\ 0, & \text { otherwise, }\end{cases}
$$

where $\alpha \leq u \leq \beta, u$ is the model value, and $\alpha$ and $\beta$ stand for the lower value and the upper value of linguistic term $\widetilde{T}$, respectively.

Given the hybrid decision matrix $\widetilde{A}=\left[\widetilde{a}_{i j}\right]_{m \times n}$, the attributes $C_{j}\left(j \in J_{1}\right)$ with linguistic assessment values may be of different granularities, and different linguistic evaluation sets would be employed [24]. Therefore, a basic linguistic evaluation set TERMSET ${ }^{B}\left(\right.$ TERMSET $^{B}=\left\{\operatorname{term}_{0}^{B}, \operatorname{term}_{1}^{B}, \ldots\right.$, $\left.\operatorname{term}_{g}^{B}\right\}$ ) is used to transform the linguistic assessment values with different granularities into the comparable form. The triangular fuzzy number $\gamma_{l}^{B}=\left(\mu_{l}^{B}, \alpha_{l}^{B}, \beta_{l}^{B}\right)$ corresponding to the linguistic term $\operatorname{term}_{i}^{B}$ is defined as follows:

$$
\gamma_{l}^{B}= \begin{cases}\alpha_{0}^{B}=0, & \\ u_{l}^{B}=\frac{l}{g-1}, & 0 \leq l \leq g-1, \\ \alpha_{l}^{B}=\frac{l-1}{g-1}, & 1 \leq l \leq g-1, \\ \beta_{l}^{B}=\frac{l+1}{g-1}, & 0 \leq l \leq g-2, \\ \beta_{g-1}^{B}=1, & \end{cases}
$$

where $\mu_{l}^{B}$ is the model value and $\alpha_{l}^{B}$ and $\beta_{l}^{B}$ stand for the lower value and the upper value of $\gamma_{l}^{B}$.

With respect to the attribute values with linguistic assessments $\widetilde{a}_{i j}\left(i=1, \ldots, m, j \in J_{1}\right)$, denote $\widetilde{a}_{i j}$ as ling $\operatorname{lin}_{i j}$. ling can be transformed into the fuzzy set over the basic linguistic evaluation set TERMSET $^{B}$ (denoted as $F_{i j}\left(\right.$ TERMSET $\left.^{B}\right)$ ):

$$
\tau: \operatorname{ling}_{i j} \longrightarrow F_{i j}\left(\text { TERMSET }^{B}\right), \quad i=1, \ldots, m, j \in J_{1},
$$

where, $F_{i j}\left(\right.$ TERMSET $\left.^{B}\right)$ is the fuzzy set over the basic linguistic evaluation set TERMSET ${ }^{B}$, as stated as follows:

$$
F_{i j}\left(\operatorname{TERMSET}^{B}\right)=\left\{\left(\operatorname{term}_{l}^{B}, \gamma_{i j, l}\right) \mid l \in[0, g], l=0, \ldots, g\right\},
$$

where

$$
\gamma_{i j, l}=\max _{y} \min \left\{\mu_{\operatorname{ling}_{i j}}(y), \mu_{\operatorname{term}_{l}^{B}}(y)\right\}
$$

where $\mu_{\operatorname{ling}_{i j}}(y)$ and $\mu_{\text {term }}^{B}(y)$ denote the membership functions of $\operatorname{ling}_{i j}$ and $\operatorname{term}_{l}^{B}$ respectively, $i=1, \ldots, m, l=0$, $\ldots, g, j \in J_{1}$.

Furthermore, the fuzzy set $F_{i j}\left(\mathrm{TERMSET}^{B}\right)$ can be transformed into a crisp value as follows:

$$
\phi\left(F_{i j}\left(\operatorname{TERMSET}^{B}\right)\right)=\frac{\sum_{l=0}^{g} l \times \gamma_{i j, l}}{g \sum_{l=0}^{g} \gamma_{i j, l}}, \quad i=1, \ldots m, j \in J_{1} .
$$

Thus, given the linguistic assessment values $\tilde{a}_{i j}$ of attributes $C_{j}\left(j \in J_{1}\right)$, by means of the operations in (3)-(6), 
linguistic term $\tilde{a}_{i j}$ is transformed into the form of crisp value, denoted as

$$
b_{i j}=\phi\left(F_{i j}\left(\operatorname{TERMSET}^{B}\right)\right), \quad i=1, \ldots m, j \in J_{1} .
$$

In the meantime, the positive ideal attribute value for attribute $C_{j}\left(j \in J_{1}\right)$ is defined as follows:

$$
\text { ideal }_{j}^{+}=\max _{1 \leq i \leq m}\left\{b_{i j}\right\}, \quad j \in J_{1} .
$$

Therefore, with respect to the attributes $C_{j}\left(j \in J_{1}\right)$ with linguistic assessment values, the grey relational coefficients between their crisp values $b_{i j}$ and the corresponding positive ideal attribute value ideal ${ }_{j}^{+}$are defined as

$$
q_{i j}=\frac{\min _{i} \min _{j}\left\{\operatorname{dis}\left(b_{i j}, \text { ideal }_{j}^{+}\right)\right\}+\rho \max _{i} \max _{j}\left\{\operatorname{dis}_{1}\left(b_{i j}, \operatorname{ideal}_{j}^{+}\right)\right\}}{\operatorname{dis}_{1}\left(b_{i j}, \text { ideal }_{j}^{+}\right)+\rho \max _{i} \max _{j}\left\{\operatorname{dis}_{1}\left(b_{i j}, \text { ideal }_{j}^{+}\right)\right\}}, \quad i=1, \ldots, m, j \in J_{1},
$$

where $\operatorname{dis}_{1}(\cdot)$ is the distance function between $b_{i j}$ and ideal ${ }_{j}^{+}$ and is defined as follows:

$$
\operatorname{Dis}_{1}\left(b_{i j}, \text { ideal }_{j}^{+}\right)=\operatorname{ideal}_{j}^{+}-b_{i j}, \quad i=1, \ldots, m, j \in J_{1} .
$$

4.1.2. Calculate the Grey Correlation Coefficients of the Attribute Values in the Form of Preference Orderings. With respect to the attributes $C_{j}\left(j \in J_{2}\right)$ with the assessment values in the form of preference orderings, denote $\tilde{a}_{i j}(i=1$, $\ldots, m, j \in J_{2}$ ) as $R_{i j}$, and $R_{i j}$ is a permutation function over the index set $\{1, \ldots, m\}$. Alternatively, $R_{i j}$ represents the position of $S_{i}$ in the preference ordering. $R_{i j}$ can be transformed into a crisp value $b_{i j}$ as follows:

$$
b_{i j}=\frac{m-R_{i j}}{m-1}, \quad i=1, \ldots, m, j \in J_{2} .
$$

In addition, regarding the attributes $C_{j}\left(j \in J_{2}\right)$ with the assessment values in the form of preference orderings, the positive ideal attribute value for attribute $C_{j}$ is defined as follows:

$$
\operatorname{ideal}_{j}^{\wedge}=\max _{1 \leq i \leq m}\left\{b_{i j}\right\}, \quad j \in J_{2} .
$$

Furthermore, with respect to the attributes $C_{j}\left(j \in J_{2}\right)$ with the assessment values in the form of preference orderings, the grey relational coefficients between their crisp values $b_{i j}$ and the corresponding positive ideal attribute value ideal ${ }_{j}^{\wedge}$ are defined as

$$
q_{i j}=\frac{\min _{i} \min _{j}\left\{\operatorname{dis}_{2}\left(b_{i j}, \operatorname{ideal}_{j}^{\wedge}\right)\right\}+\rho \max _{i} \max _{j}\left\{\operatorname{dis}_{2}\left(b_{i j}, \operatorname{ideal}_{j}^{\wedge}\right)\right\}}{\operatorname{dis}_{2}\left(b_{i j}, \operatorname{ideal}_{j}^{\wedge}\right)+\rho \max _{i} \max _{j}\left\{\operatorname{dis}_{2}\left(b_{i j}, \operatorname{ideal}_{j}^{\wedge}\right)\right\}}, \quad i=1, \ldots, m, j \in J_{2},
$$

where $\operatorname{dis}_{2}(\cdot)$ is the distance function between $b_{i j}$ and ideal ${ }_{j}^{\wedge}$ and defined as follows:

$$
\operatorname{Dis}_{2}\left(b_{i j}, \text { ideal }_{j}^{\wedge}\right)=\operatorname{ideal}_{j}^{\wedge}-b_{i j}, \quad i=1, \ldots, m, j \in J_{2} .
$$

4.1.3. Calculate the Grey Correlation Coefficients of the Interval Attribute Values. With respect to the attributes $C_{j}$ $\left(j \in J_{3}\right)$ with interval assessment values, denote $\widetilde{a}_{i j}$ as interval numbers $\left[a_{i j}^{L}, a_{i j}^{U}\right]\left(i=1, \ldots, m, j \in J_{3}\right) .\left[a_{i j}^{L}, a_{i j}^{U}\right]$ can be transformed into the benefit type, denoted as $\left[b_{i j}^{L}, b_{i j}^{U}\right]$ $\left(i=1, \ldots, m, j \in J_{3}\right)$.

Definition 2. Given interval attribute values $\widetilde{b}_{i \dot{j}}$ and $\widetilde{b}_{k j}$ for $C_{j}, i, k=1, \ldots, m, j \in J_{3}$, the distance between $\tilde{b}_{i j}$ and $\tilde{b}_{k j}$ is defined as

$$
\operatorname{dis}_{3}\left(\widetilde{b}_{i j}, \widetilde{b}_{k j}\right)=\frac{\sqrt{2}}{2} \sqrt{\left(b_{i j}^{L}-b_{k j}^{L}\right)^{2}+\left(b_{i j}^{U}-b_{k j}^{U}\right)^{2}}, \quad i, k=1, \ldots, m, j \in J_{3} .
$$

Definition 3. With respect to the attributes $C_{j}\left(j \in J_{3}\right)$ with interval assessment values, after the interval attribute values $\left[a_{i j}^{L}, a_{i j}^{U}\right]\left(i=1, \ldots, m, j \in J_{3}\right)$ are transformed into the benefit type $\left[b_{i j}^{L}, b_{i j}^{U}\right]$, the positive ideal attribute value for attribute $C_{j}$ is defined as follows:

$$
\text { ideal }_{j}^{*}=\left[\text { ideal }_{j}^{* L}, \text { ideal }_{j}^{* U}\right], \quad j \in J_{3},
$$

where

$$
\begin{array}{ll}
\text { ideal }_{j}^{* L}=\max _{1 \leq i \leq m}\left\{b_{i j}^{L}\right\}, & j \in J_{3}, \\
\text { ideal }_{j}^{* U}=\max _{1 \leq i \leq m}\left\{b_{i j}^{U}\right\}, & j \in J_{3} .
\end{array}
$$

Definition 4. Given the hybrid decision matrix $\widetilde{A}=\left[\widetilde{a}_{i j}\right]_{m \times n}$, with respect to the attributes $C_{j}\left(j \in J_{3}\right)$ with interval assessment values, the grey relational coefficients between 
$\left[b_{i j}^{L}, b_{i j}^{U}\right]$ and the corresponding positive ideal attribute value ideal $_{j}^{*}$ are defined as

$$
q_{i j}=\frac{\min _{i} \min _{j}\left\{\operatorname{dis}_{3}\left(\widetilde{b}_{i j}, \operatorname{ideal}_{j}^{*}\right)\right\}+\rho \max _{i} \max _{j}\left\{\operatorname{dis}_{3}\left(\widetilde{b}_{i j}, \text { ideal }_{j}^{*}\right)\right\}}{\operatorname{dis}_{3}\left(\widetilde{b}_{i j}, \text { ideal }_{j}^{*}\right)+\rho \max _{i} \max _{j}\left\{\operatorname{dis}_{3}\left(\widetilde{b}_{i j}, \text { ideal }_{j}^{*}\right)\right\}}, \quad i=1, \ldots, m, j \in J_{3},
$$

where $\operatorname{dis}_{3}(\cdot)$ is the distance function between two interval numbers as defined in (15) and $\rho$ is the parameter which usually has a value of 0.5 .

4.2. Determine Attribute Weights. After the hybrid decision matrix $\widetilde{A}=\left[\widetilde{a}_{i j}\right]_{m \times n}$ is transformed into $Q=\left[q_{i j}\right]_{m \times n}$, in this section, a deviation maximization model is proposed firstly to determine the attribute weights and integrate them with those derived by the entropy method.

\subsubsection{The Proposed Deviation Maximization Model}

Definition 5. Given the normalized and beneficial decision matrix $Q=\left(q_{i j}\right)_{m \times n}$, for attribute $C_{j}$, the weighted distance between alternative $S_{i}$ and all other alternatives is defined as

$$
D_{i j}(W)=\sum_{k=1}^{m} \operatorname{dev}\left(q_{i j}, q_{k j}\right) w_{j}, \quad i=1, \ldots, m, j=1, \ldots, n,
$$

where $W=\left(w_{1}, w_{2}, \ldots, w_{n}\right)$ is the weight vector of the attributes, and $\operatorname{dev}(\cdot)$ is the difference function between two attribute values and is defined as follows:

$$
\operatorname{dev}\left(q_{i j}, q_{k j}\right)=\left|q_{i j}-q_{k j}\right|, \quad i, k=1, \ldots, m, j=1, \ldots, n .
$$

Definition 6. Given the normalized and beneficial decision matrix $Q=\left(q_{i j}\right)_{m \times n}$, for attribute $C_{j}$, the weighted distance between all alternatives and others is defined as

$$
D_{j}(W)=\sum_{i=1}^{m} D_{i j}(W)=\sum_{i=1}^{m} \sum_{k=1}^{m} \operatorname{dev}\left(q_{i j}, q_{k j}\right) w_{j}, \quad j=1, \ldots, n .
$$

It can be seen that $D_{j}(w)$ denotes the weighted distances among all alternatives for attribute $C_{j}, j=1, \ldots, n$. Assuming that all the alternatives are equally competitive and there is no preference between them, the optimal weight vector $W\left(W=\left(w_{1}, w_{2}, \ldots, w_{n}\right)\right)$ of the attributes should maximize the weighted distances among all alternatives across all the attributes. Therefore, the following deviation maximization model is set up to determine the attribute weights:

$$
\max D(w)=\sum_{j=1}^{n} D_{j}(w)=\sum_{i=1}^{m} \sum_{j=1}^{n} \sum_{k=1}^{m} \operatorname{dev}\left(q_{i j}, q_{k j}\right) w_{j},
$$

s.t.

$$
\begin{array}{r}
\sum_{j=1}^{n} w_{j}^{2}=1, \quad j=1, \ldots, n, \\
w_{j} \geq 0, \quad j=1, \ldots, n .
\end{array}
$$

Theorem 1. The optimal solution to model $(22 a)-(22 c)$ is

$$
w_{j}=\frac{\sum_{i=1}^{m} \sum_{k=1}^{m} \operatorname{dev}\left(q_{i j}, q_{k j}\right)}{\sqrt{\sum_{j=1}^{n}\left(\sum_{i=1}^{m} \sum_{k=1}^{m} \operatorname{dev}\left(q_{i j}, q_{k j}\right)\right)^{2}}}, \quad j=1, \ldots, n .
$$

Proof:

The following Lagrange function is constructed:

$$
L(W, \lambda)=\sum_{i=1}^{m} \sum_{j=1}^{n} \sum_{k=1}^{m} \operatorname{dev}\left(q_{i j}, q_{k j}\right) w_{j}+\frac{\lambda}{2}\left(\sum_{j=1}^{n} w_{j}^{2}-1\right) \text {, }
$$

where $\lambda$ is the Lagrange multiplier.

Let $\partial L / \partial w_{j}=0$ and $\partial L / \partial \lambda=0$, the following equation can be obtained:

$$
\begin{aligned}
\sum_{i=1}^{m} \sum_{k=1}^{m} \operatorname{dev}\left(q_{i j}, q_{k j}\right)+\lambda w_{j} & =0, \quad j=1, \ldots, n, \\
\sum_{j=1}^{n} w_{j}^{2} & =1 .
\end{aligned}
$$

By solving the equations composed of (25a) and (25b), the following can be obtained:

$$
\begin{aligned}
& \lambda=-\left[\sum_{j=1}^{n}\left(\sum_{i=1}^{m} \sum_{k=1}^{n} \operatorname{dev}\left(q_{i j}, q_{k j}\right)\right)\right]^{1 / 2}, \\
& w_{j}=\frac{\sum_{i=1}^{m} \sum_{k=1}^{m} \operatorname{dev}\left(q_{i j}, q_{k j}\right)}{\sqrt{\sum_{j=1}^{n}\left(\sum_{i=1}^{m} \sum_{k=1}^{m} \operatorname{dev}\left(q_{i j}, q_{k j}\right)\right)^{2}}}, \quad k=1, \ldots, m .
\end{aligned}
$$


Furthermore, normalize the weights given by (26b), and the weight of attribute $C_{j}(j=1, \ldots, n)$ is obtained:

$$
w_{j}^{1}=\frac{\sum_{i=1}^{m} \sum_{k=1}^{m} \operatorname{dev}\left(q_{i j}, q_{k j}\right)}{\sum_{j=1}^{n}\left(\sum_{i=1}^{m} \sum_{k=1}^{m} \operatorname{dev}\left(q_{i j}, q_{k j}\right)\right)}, \quad j=1, \ldots, n .
$$

Thus, weight vector $W^{1}\left(W^{1}=\left(w_{1}^{1}, w_{2}^{1}, \ldots, w_{n}^{1}\right)\right)$ of the attributes can be obtained based on maximizing the weighted distances among all alternatives across the attributes.

4.2.2. Entropy Method. Based on the normalized and beneficial decision matrix $Q=\left(q_{i j}\right)_{m \times n}$, the attribute weights can be calculated by means of the entropy method [25]:

(a) Calculate the proportions of attributes in matrix $Q$ : Given the normalized and beneficial decision matrix $Q=\left(q_{i j}\right)_{m \times n}$, for attribute $C_{j}$, the ratio of $q_{i j}$ to the sum of all elements in the same column in $Q$ is calculated as

$$
Z_{\mathrm{ij}}=\frac{q_{i j}}{\sum_{l=1}^{n} q_{l j}}, \quad i=1, \ldots, m, j=1, \ldots, n .
$$

(b) Calculate the information entropy of the attributes: The information entropy of the attributes can be calculated as follows:

$$
E_{j}=-k \sum_{i=1}^{m} Z_{i j} \ln z_{i j}, \quad j=1, \ldots, n,
$$

where $k$ is the adjustment coefficient and $k=1 / \ln n$.

(c) Calculate the redundancy of information entropy:

$$
e_{j}=1-E_{j}, \quad j=1, \ldots, n .
$$

(d) Calculate the weights of attributes:

Based on the information entropy of the attributes, their weights can be calculated as follows:

$$
W_{j}=\frac{e_{j}}{\sum_{j=1}^{n} e_{j}}, \quad j=1, \ldots, n .
$$

Denote $W^{2}=\left(w_{1}^{2}, w_{2}^{2}, \ldots, w_{n}^{2}\right)$ as the weight vector of the attributes obtained by means of the entropy method.

4.2.3. Determine Comprehensive Attribute Weights. Based on the attribute weight vector $W^{1}$ calculated by the deviation maximization model (22a)-(22c) and the attribute weight vector $W^{2}$ obtained by the Entropy method, the comprehensive attribute weight vector based on the decision matrix $Q$ is calculated in the following:

$$
W^{o}=0.5 W^{1}+0.5 W^{2} \text {. }
$$

4.3. Calculate the Overall Values of Suppliers. The overall values of supplier $S_{i}$ can be obtained by means of the weighted sum method as follows:

$$
\text { overall }_{i}=\sum_{j=1}^{n} w_{j}^{o} q_{i j}, \quad i=1, \ldots, m .
$$

All the suppliers can be ranked descendingly according to their overall values calculated in formula (33).

\section{Illustrations}

In the course of supplier selection, the determination of assessment attributes is the first step. The selection of attributes is reviewed in [9]. In this study, service level $\left(C_{1}\right)$, degree of informatization $\left(C_{2}\right)$, profitability $\left(C_{3}\right)$, level of quality $\left(C_{4}\right)$, and level of risk $\left(C_{5}\right)$ are adopted in evaluating and selecting four suppliers (i.e., $S_{i}, i=1,2,3$, and 4 ) in B2B e-commerce environment. Because of the uncertainty and fuzziness in B2B e-commerce environment, for the attributes of service level $\left(C_{1}\right)$ and level of risk $\left(C_{5}\right)$, preference orderings are used.

For the attributes of degree of informatization $\left(C_{2}\right)$ and level of quality $\left(C_{4}\right)$, linguistic terms are used to assess the supplier performances. For the attribute of profitability $C_{3}$, interval numbers are employed. For the sake of simplicity, the linguistic term set \{"very poor," "poor," "fair," "good," and "very good" $\}$ is employed for both attributes of degree of informatization $\left(C_{2}\right)$ and level of quality $\left(C_{4}\right)$, and is same as the basic linguistic term set TERMSET ${ }^{B}$ in this study. Details of the assessment information of the suppliers $\left(S_{i}, i=1,2,3\right.$, 4) against the attributes are stated in Table 1.

Firstly, with respect to attribute $C_{1}$ of the service level, the assessment information of the suppliers in Table 1 is normalized as $\left(b_{11}, b_{21}, b_{31}, b_{41}\right)^{\mathrm{T}}=(1,0,0.3333,0.6667)^{\mathrm{T}}$ and is further transformed as $\left(q_{11}, q_{21}, q_{31}, q_{41}\right)^{\mathrm{T}}=(1,0.3333$, $0.4286,0.6)^{\mathrm{T}}$, by calculating the grey relational coefficients between their crisp values $b_{i 1}(i=1,2,3,4)$ and the corresponding positive ideal attribute value.

Secondly, with respect to attribute $C_{2}$ of degree of informatization, the linguistic assessment information of the suppliers in Table 1 is normalized as $\left(b_{12}, b_{22}, b_{32}, b_{42}\right)^{\mathrm{T}}=(0.9167,0.25,0.5$, $0.75)^{\mathrm{T}}$ and is further transformed as $\left(q_{12}, q_{22}, q_{32}, q_{42}\right)^{\mathrm{T}}=(1,0.4$, $0.5714,1)^{\mathrm{T}}$.

Thirdly, with respect to attribute $C_{3}$ of profitability, the interval assessment information of the suppliers in Table 1 is normalized as

$$
\left(\begin{array}{ll}
b_{13}^{l} & b_{13}^{u} \\
b_{23}^{l} & b_{23}^{u} \\
b_{33}^{l} & b_{33}^{u} \\
b_{43}^{l} & b_{43}^{u}
\end{array}\right)=\left(\begin{array}{cc}
0.75 & 1 \\
0 & 0.25 \\
0.5 & 0.75 \\
0.25 & 0.5
\end{array}\right),
$$

and it is further transformed as $\left(q_{13}, q_{23}, q_{33}, q_{43}\right)^{\mathrm{T}}=(0.5836$, $0.5182,1,0.6940)^{\mathrm{T}}$.

Fourthly, with respect to attribute $C_{4}$ of level of quality, the linguistic assessment information of the suppliers in Table 1 is normalized as $\left(b_{14}, b_{24}, b_{34}, b_{44}\right)$ $\mathrm{T}=(0.5,0.0833,0.75,0.9167)^{\mathrm{T}}$ and is further transformed as $\left(q_{14}, q_{24}, q_{34}, q_{44}\right)^{\mathrm{T}}=(0.5,0.3333,0.7143,1)^{\mathrm{T}}$. Fifthly, with respect to attribute $C_{5}$ of level of risk, the assessment information of the suppliers in Table 1 is normalized into 
TABLE 1: The assessment information of the suppliers.

\begin{tabular}{cccccc}
\hline & Service level $C_{1}$ & Degree of informatization $C_{2}$ & Profitability $C_{3}$ & Level of quality $C_{4}$ & Level of risk $C_{5}$ \\
\hline$S_{1}$ & 1 & Poor & {$[70,80]$} & Fair & 2 \\
$S_{2}$ & 4 & Very poor & {$[40,50]$} & Poor & 1 \\
$S_{3}$ & 3 & Fair & {$[60,70]$} & Very good & Good \\
$S_{4}$ & 2 & Very good & {$[50,60]$} & 3 \\
\hline
\end{tabular}

$\left(b_{15}, b_{25}, b_{35}, b_{45}\right)^{\mathrm{T}}=(0.6667,1,0,0.3333)^{\mathrm{T}}$ and is further transformed as $\left(q_{15}, q_{25}, q_{35}, q_{45}\right)^{\mathrm{T}}=(0.6,1,0.3333$, $0.4286)^{\mathrm{T}}$.

Based on the above calculation, the single-point value decision matrix $Q$ is obtained as

$$
Q=\left(\begin{array}{lllll}
1.0000 & 1.0000 & 0.5836 & 0.5000 & 0.6000 \\
0.3333 & 0.4000 & 0.5182 & 0.3333 & 1.0000 \\
0.4286 & 0.5714 & 1.0000 & 0.7143 & 0.3333 \\
0.6000 & 1.0000 & 0.6940 & 1.0000 & 0.4286
\end{array}\right)
$$

Furthermore, based on the normalized decision matrix $Q$, the attribute weight vector can be calculated by the deviation maximization model (22a) $-(22 \mathrm{c})$ as $W^{1}=(0.2100,0.2155$, $0.1504,0.2141$, and 0.2100$)$. In the meantime, the attribute weight vector can also be obtained by the entropy weight method as $W^{2}=(0.2120,0.1970,0.1746,0.2044$, and 0.2120). Thus, the comprehensive attribute weight vector based on the decision matrix $Q$ is calculated as $W^{\mathrm{o}}=(0.2110,0.2062$, $0.1625,0.2092$, and 0.2110$)$. Accordingly, the overall values of the suppliers can be obtained as follows: overall $1=0.7432$, overall $_{2}=0.5177$, overall $3=0.6295$, and overall $4=0.7452$. Finally, the ranking of the suppliers is $S_{4}>S_{1}>S_{3}>S_{2}$.

\section{Conclusions}

This paper proposes an approach to evaluating and selecting the manufacturing suppliers in $\mathrm{B} 2 \mathrm{~B}$ e-commerce environment, where linguistic terms, preference orderings, and interval numbers are employed to present their fuzzy performances. After the hybrid decision matrix is normalized, the attribute weights are determined by means of proposing the deviation maximization model and the entropy method.

The merits of the proposed approach lie in three aspects. The first one is to express the uncertainty of the suppliers' performances by means of the appropriate and the easiest ways, i.e., linguistic terms, preference orderings, and interval numbers. The second one is to propose the methods of normalizing the hybrid decision matrix by calculating the grey correlation coefficients of attribute values with the ideal values of attributes. The third one is to determine the attribute weights by means of the deviation maximization model and the entropy method based on normalized decision matrix. This paper enables to express the suppliers' performance information in the easiest ways and accurately, especially in fuzzy or uncertain decision environment. Compared with the current research, the proposed approach has more universal significance and practical application prospects.

\section{Data Availability}

The data used to support the findings of this study are available from the corresponding author upon request.

\section{Conflicts of Interest}

The authors declare that they have no conflicts of interest.

\section{Acknowledgments}

This work was supported by the Science and Technology Agency of Liaoning Province under the Grant of the Natural Science Foundation of Liaoning Province (2013020022)

"Hybrid multicriteria group decision-making with various forms of information expression."

\section{References}

[1] D. Maffin and P. Braiden, "Manufacturing and supplier roles in product development," International Journal of Production Economics, vol. 69, no. 2, pp. 205-213, 2001.

[2] G. Svensson, T. Mysen, and J. Payan, "Balancing the sequential logic of quality constructs in manufacturing-supplier relationships-causes and outcomes," Journal of Business Research, vol. 63, no. 11, pp. 1209-1214, 2010.

[3] M. J. Sáenz, D. Knoppen, and E. M. Tachizawa, "Building manufacturing flexibility with strategic suppliers and contingent effect of product dynamism on customer satisfaction," Journal of Purchasing and Supply Management, vol. 24, no. 3, pp. 238-246, 2018.

[4] T.-C. Chu and R. Varma, "Evaluating suppliers via a multiple levels multiple criteria decision making method under fuzzy environment," Computers \& Industrial Engineering, vol. 62, no. 2, pp. 653-660, 2012.

[5] L. Xie, J. Ma, and H. Han, "Implications of stochastic demand and manufacturers' operational mode on retailer's mixed bundling strategy and its complexity analysis," Applied Mathematical Modelling, vol. 55, no. 1, pp. 484-501, 2018.

[6] J. Ma and L. Xie, "The stability analysis of the dynamic pricing strategy for bundling goods: a comparison between simultaneous and sequential pricing mechanism," Nonlinear Dynamics, vol. 95, no. 2, pp. 1147-1164, 2019.

[7] L. A. Zadeh, "A computational approach to fuzzy quantifiers in natural languages," Computational Linguistics, vol. 9, no. 1, pp. 149-184, 1983.

[8] L. F. D. O. M. Santos, L. Osiro, and R. H. P. Lima, "A model based on 2-tuple fuzzy linguistic representation and Analytic Hierarchy Process for supplier segmentation using qualitative and quantitative criteria," Expert Systems with Applications, vol. 79, pp. 53-64, 2017.

[9] P. Sureeyatanapas, K. Sriwattananusart, T. Niyamosoth, W. Sessomboon, and S. Arunyanart, "Supplier selection towards uncertain and unavailable information: an extension of 
TOPSIS method," Operations Research Perspectives, vol. 5, pp. 69-79, 2018.

[10] C.-N. Liao and H.-P. Kao, "An integrated fuzzy TOPSIS and MCGP approach to supplier selection in supply chain management," Expert Systems with Applications, vol. 38, no. 9, pp. 10803-10811, 2011.

[11] F. R. L. Junior, L. Osiro, and L. C. R. Carpinetti, "A comparison between fuzzy AHP and fuzzy TOPSIS methods to supplier selection," Applied Soft Computing, vol. 21, no. 5, pp. 194-209, 2014.

[12] A. Azizi, D. O. Aikhuele, and F. S. Souleman, "A fuzzy TOPSIS model to rank automotive suppliers," Procedia Manufacturing, vol. 2, pp. 159-164, 2015.

[13] A. Kokangul and Z. Susuz, "Integrated analytical hierarch process and mathematical programming to supplier selection problem with quantity discount," Applied Mathematical Modelling, vol. 33, no. 3, pp. 1417-1429, 2009.

[14] N. R. Ware, S. P. Singh, and D. K. Banwet, "A mixed-integer non-linear program to model dynamic supplier selection problem," Expert Systems with Applications, vol. 41, no. 2, pp. 671-678, 2014.

[15] H. Mirzaee, B. Naderi, and S. H. R. Pasandideh, "A preemptive fuzzy goal programming model for generalized supplier selection and order allocation with incremental discount," Computers \& Industrial Engineering, vol. 122, pp. 292-302, 2018.

[16] C. L. Hwang and K. Yoon, Multiple Attribute Decision Making: Methods and Applications, Springer-Verlag, New York, NY, USA, 1981.

[17] C. C. Lee and C. Ou-Yang, "A neural networks approach for forecasting the supplier's bid prices in supplier selection negotiation process," Expert Systems with Applications, vol. 36, no. 2, pp. 2961-2970, 2009.

[18] A. F. Güneri, T. Ertay, and A. Yücel, "An approach based on ANFIS input selection and modeling for supplier selection problem," Expert Systems with Applications, vol. 38, no. 12, pp. 14907-14917, 2011.

[19] C. Bai and J. Sarkis, "Integrating sustainability into supplier selection with grey system and rough set methodologies," International Journal of Production Economics, vol. 124, no. 1, pp. 252-264, 2010.

[20] D. Golmohammadi and M. Mellat-Parast, "Developing a grey-based decision-making model for supplier selection," International Journal of Production Economics, vol. 137, no. 2, pp. 191-200, 2012.

[21] M. S. Memon, Y. H. Lee, and S. I. Mari, "Group multi-criteria supplier selection using combined grey systems theory and uncertainty theory," Expert Systems with Applications, vol. 42, no. 21, pp. 7951-7959, 2015.

[22] B. S. Ahn and K. S. Park, "Comparing methods for multiattribute decision making with ordinal weights," Computers \& Operations Research, vol. 35, no. 5, pp. 1660-1670, 2008.

[23] J. Wang and S. Zionts, "Using ordinal data to estimate cardinal values," Journal of Multi-Criteria Decision Analysis, vol. 22, no. 3-4, pp. 185-196, 2015.

[24] F. Herrera, L. Martínez, and P. J. Sánchez, "Managing nonhomogeneous information in group decision making," $E$ ropean Journal of Operational Research, vol. 166, no. 1, pp. 115-132, 2005.

[25] Y. Ji, G. H. Huang, and W. Sun, "Risk assessment of hydropower stations through an integrated fuzzy entropyweight multiple criteria decision making method: a case study of the Xiangxi River," Expert Systems with Applications, vol. 42, no. 12, pp. 5380-5389, 2015. 